\title{
Connaissances et usages de Cola millenii K. Schum. (Malvaceae) en zones guinéenne et soudano-guinéenne au Bénin
}

Iboukoun Fidèle LAWIN ${ }^{1,2}$ Towanou HouÈTCHÉGNON ${ }^{2}$ Adandé Belarmain FANDOHAN ${ }^{1,3,4}$ Valère Kolawolé SALAKO ${ }^{4}$ Achille Ephrem Assogbadjo ${ }^{2}$ Christine Adjokè OuINSAVI ${ }^{1}$

\section{${ }^{1}$ Université de Parakou} Faculté d'agronomie Laboratoire d'études et de recherches forestières (LERF) BP 123, Parakou Bénin

2 Université d’Abomey-Calavi Faculté des sciences agronomiques Laboratoire d'écologie appliquée (LEA) 01 BP 526, Cotonou

\section{Bénin}

${ }^{3}$ Université nationale d'agriculture École de foresterie et ingénierie du bois Unité de recherche en foresterie, agroforesterie et biogéographie BP 43, Kétou

Bénin

${ }^{4}$ Université d'Abomey-Calavi Faculté des sciences agronomiques Laboratoire de biomathématiques et d'estimations forestières (LABEF) 04 BP 1525, Cotonou Bénin

\section{Auteur correspondant /} Corresponding author: Iboukoun Fidèle Lawin ifilawin@yahoo.fr

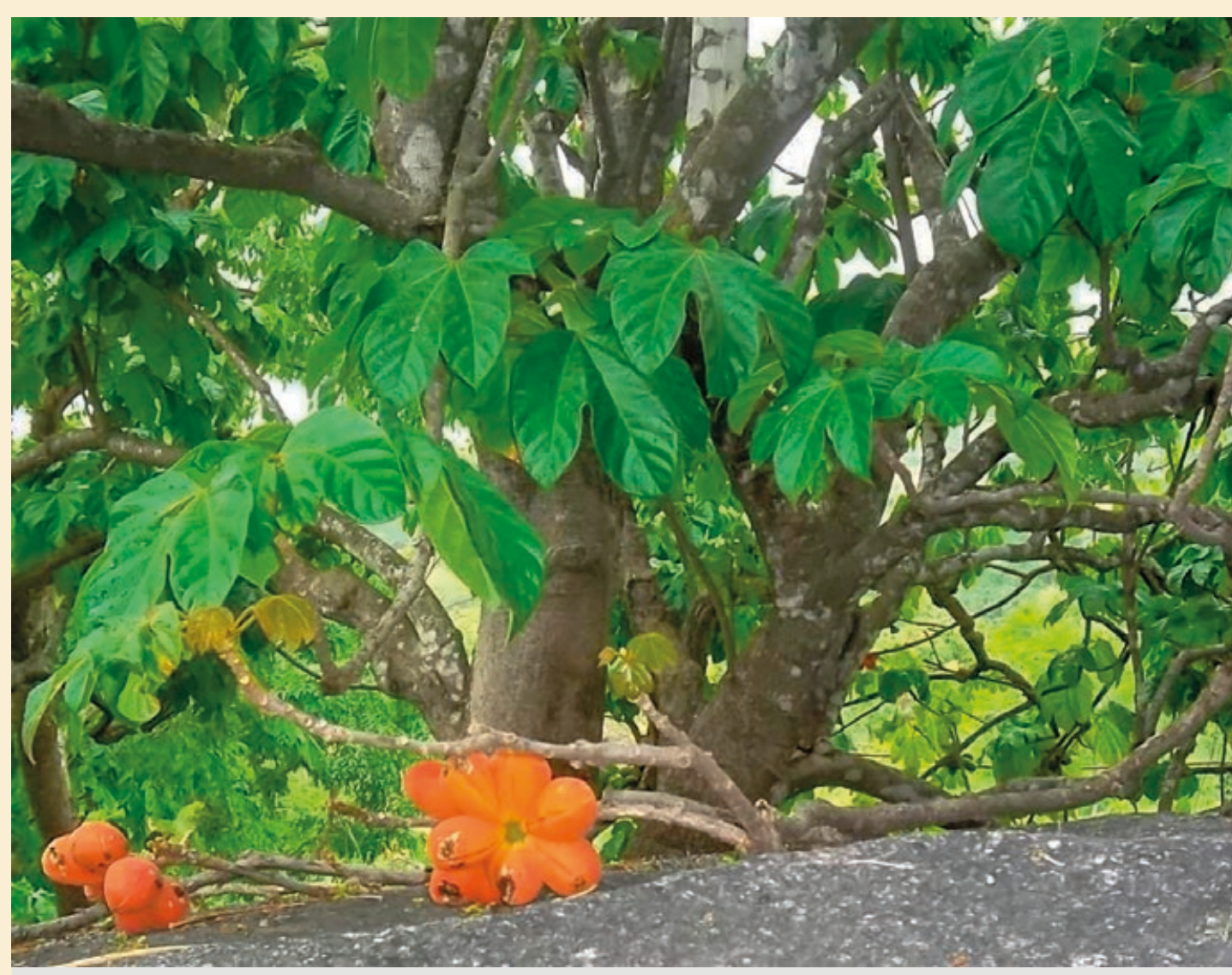

Photo 1.

Individu adulte de Cola millenii : feuilles digitées et fruits. Photo I. F. Lawin.

Doi : 10.19182/bft2019.339.a31716 - Droit d'auteur (c) 2018, Bois et Forêts des Tropiques - (c) Cirad - Date de soumission : 21 mars 2018 ; date d'acceptation : 3 septembre 2018 ; date de publication : 30 janvier 2019.

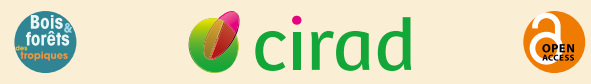

(c) $(1) \Theta$
Citer l'article / To cite the article

Lawin I. F., Houètchégnon T., Fandohan A. B., Salako V. K., Assogbadjo A. E., Ouinsavi C. A., 2019. Connaissances et usages de Cola millenii K. Schum. (Malvaceae) en zones guinéenne et soudano-guinéenne au Bénin. Bois et Forêts des Tropiques, 339: 61-74. Doi : https://doi.org/10.19182/ bft2019.339.a31716 


\section{RÉSUMÉ}

\section{Connaissances et usages de Cola millenii K. Schum. (Malvaceae) en zones guinéenne et soudano-guinéenne au Bénin}

Bien que plusieurs fruitiers sauvages ont été étudiés au Bénin, d'autres, dont Cola millenii K. Schum., y demeurent peu documentés. Cette étude avait pour objectif d'inventorier les connaissances ethnobotaniques de $C$. millenii par les groupes socioculturels béninois. Des entretiens individuels structurés ont été menés auprès de 1009 personnes. La fréquence relative de citation et l'indice d'efficacité ont été utilisés pour apprécier respectivement l'importance relative de chaque utilisation et le niveau d'efficacité de la plante dans le traitement d'une maladie donnée. $C$. millenii relève de huit catégories d'usage. L'usage alimentaire de la pulpe du fruit est le plus pratiqué. Le paludisme est la maladie la plus citée. Globalement, l'espèce serait très efficace dans le traitement du paludisme, de l'ictère, des maux de ventre, de l'hypertension, des courbatures, de la fatigue, de la fièvre et du vertige ; moyennement efficace dans le traitement de l'asthme, des troubles liés à la grossesse et de l'enflure des pieds ; mais peu efficace contre l'oligospermie. La feuille est la partie la plus utilisée et la décoction est le mode de préparation le plus employé dans le traitement des maladies. Les Anii et Chabè préfèrent l'usage alimentaire de l'espèce tandis que son usage à des fins magiques et comme brosse végétale ou bois de service est privilégié par les Adja et Kotafon. Par contre, les Aïzo l'utilisent plus à des fins médicinales, et son usage comme bois d'œuvre est plus important pour les Mahi. Les feuilles et les fruits sont les parties les plus commercialisées. Malgré son importance, l'espèce subit diverses pressions et bénéficie très peu de mesures de conservation. Des recherches futures devraient se pencher sur sa sylviculture pour assurer sa domestication.

Mots-clés : Cola millenii, ethnobotanique, fruitiers sauvages, groupes socioculturels, Afrique de l'Ouest.

\section{ABSTRACT}

\begin{abstract}
Knowledge and uses of Cola millenii K. Schum. (Malvaceae) in the Guinean and Sudano-Guinean zones of Benin
\end{abstract}

Although a number of wild fruit trees in Benin have been studied, others, such as Cola millenii K. Schum., are still relatively undocumented. The aim of this study was to collect ethnobotanical knowledge on C. millenii among socio-cultural groups in Benin. Individual structured interviews were conducted with 1009 people. Relative frequency of citation and the efficiency index were used to assess the relative importance of each use and the degree of effectiveness of the plant in treating given ailments. C. millenii falls into eight different use categories. Most frequently cited is use of the fruit pulp for food, and malaria is the most frequently cited ailment. Overall, the species is considered to be very effective against malaria, jaundice, stomach pains, high blood pressure, stiff joints, fatigue, fever and vertigo; fairly effective against asthma, pregnancy-related disorders and swollen feet; and not effective to treat a low sperm count. The leaves are most frequently used to treat ailments, mainly as decoctions. The Anii and Chabè use the species preferentially for food, while the Adja and Kotafon mainly use it for magical purposes, to make brushes and as construction wood. It is used most frequently for medicinal purposes by the Aïzo, while the Mahi consider it to be most useful for construction. Trade is mainly in the leaves and fruit. Despite its importance, the species is under pressure in various ways and inadequately protected. Future studies need to focus on sylvicultural techniques with a view to domesticating the species.

Keywords: Cola millenii, ethnobotany, wild fruit trees, socio-cultural groups, West Africa.

\section{RESUMEN}

\author{
Conocimiento y usos de Cola millenii \\ K. Schum. (Malvaceae) en las zonas \\ guineana y sudanoguineana \\ en Benín
}

Aunque se hayan estudiado varios frutales silvestres en Benín, otros, entre los cuales Cola millenii K. Schum., todavía están poco documentados. Este estudio tenía como objetivo inventariar los conocimientos etnobotánicos de C. millenii que tienen los grupos socioculturales benineses. Se llevaron a cabo entrevistas individuales estructuradas a 1009 personas. La frecuencia relativa de citación y el índice de eficacia se utilizaron para apreciar respectivamente la importancia relativa de cada utilización y el nivel de eficacia de la planta en el tratamiento de una enfermedad determinada. C. millenii tiene ocho categorías de uso. El uso alimenticio de la pulpa del fruto es el más practicado. El paludismo es la enfermedad más citada. Globalmente, la especie sería muy eficaz en el tratamiento de paludismo, ictericia, dolores de barriga, hipertensión, agujetas, cansancio, fiebre y vértigos; medianamente eficaz en el tratamiento de asma, trastornos relacionados con el embarazo e hinchazón de los pies; pero poco eficaz contra la oligospermia. La hoja es la parte más utilizada y la decocción es la forma de preparación más empleada en el tratamiento de las enfermedades. Los Anii y Chabè prefieren el uso alimenticio de la especie mientras que su uso con fines mágicos y como cepillo vegetal o madera de servicio es preferente para los Adja y Kotafon. En cambio, los Aïzo lo utilizan más con fines medicinales, y su uso como madera de obra es más importante para los Mahi. Las hojas y los frutos son las partes más comercializadas. A pesar de su importancia, la especie sufre diferentes presiones y dispone a penas de medidas de conservación. Las futuras investigaciones deberían inclinarse hacia su silvicultura para asegurar su domesticación.

Palabras clave: Cola millenii, etnobotánica, frutales silvestres, grupos socioculturales, África Occidental. 


\section{Introduction}

Les communautés rurales africaines possèdent des connaissances traditionnelles sur la valeur et les propriétés de nombreuses espèces végétales (Moupela et al., 2011). Les politiques de gestion des ressources forestières ne sauraient être durables que si elles intègrent les valeurs sociales, culturelles et économiques que les communautés locales leur associent. Dans ce sens, les études ethnobotaniques apparaissent comme une bonne approche pour comprendre, dans une région donnée, les utilisations ainsi que les perceptions socioculturelles des ressources forestières par les populations locales (Agbogidi, 2010). Les connaissances endogènes, composante essentielle de la conservation de la biodiversité (Pilgrim etal., 2007), importent aussi bien dans l'amélioration de la vie quotidienne des populations que dans la prise de décision pour la gestion des ressources (Campos et Ehringhaus, 2003). Les études ethnobotaniques permettent de recenser les utilisations locales des espèces végétales. Ces connaissances viennent à la base de toutes approches visant à proposer des solutions efficaces face aux menaces exercées sur ces espèces au niveau des communautés locales (Akpavi et al., 2011).

L'explosion démographique menace de disparition des reliques forestières, avec les espèces résiduelles qu'elles abritent, fournisseuses de produits forestiers non ligneux. Il apparaît donc urgent de documenter la connaissance des communautés rurales à l'égard de ces espèces, et d'envisager leur sauvegarde (Djaha et Gnahoua, 2014). Au nombre des fruitiers sauvages, figure Cola millenii K. Schum, utilisé en médecine traditionnelle et dans l'alimentation au Nigeria (Odugbemi, 2006), en Côte d'Ivoire (Kouamé et al., 2008) et au Togo (Denou et al., 2016). Au Bénin, en plus de son utilisation médicinale (Akoègninou et al., 2006 ; Adomou et al., 2012) et alimentaire (Assogbadjo et al., 2012), l'espèce est utilisée comme bois énergie et bois d'œuvre (Dossou et al., 2012). Ces études, centrées sur l'importance socioculturelle ou la valeur d'usage de cette espèce, renseignent peu la diversité effective des usages pourtant observée selon les groupes socioculturels (Case et al., 2005 ; Atakpama et al. 2012 ; Franco et al., 2014 ; Assogba et al., 2017). C'est dans la perspective de valoriser et d'utiliser durablement l'espèce que cette étude a été initiée et visait à inventorier les connaissances ethnobotaniques de C. millenii par les groupes socioculturels du Bénin.

\section{Matériel et méthodes}

\section{Milieu d'étude}

L'étude s'est déroulée au sein de l'aire de répartition de C. millenii au Bénin, soit sept districts phytogéographiques correspondant aux zones guinéenne et soudano-guinéenne. Il s'agit des quatre districts phytogéographiques (Côtier, Pobè, Vallée de l'Ouémé, Plateau) de la zone guinéenne et des trois districts phytogéographiques (Zou, Bassila, Borgou sud) de la zone soudano-guinéenne (figure 1).

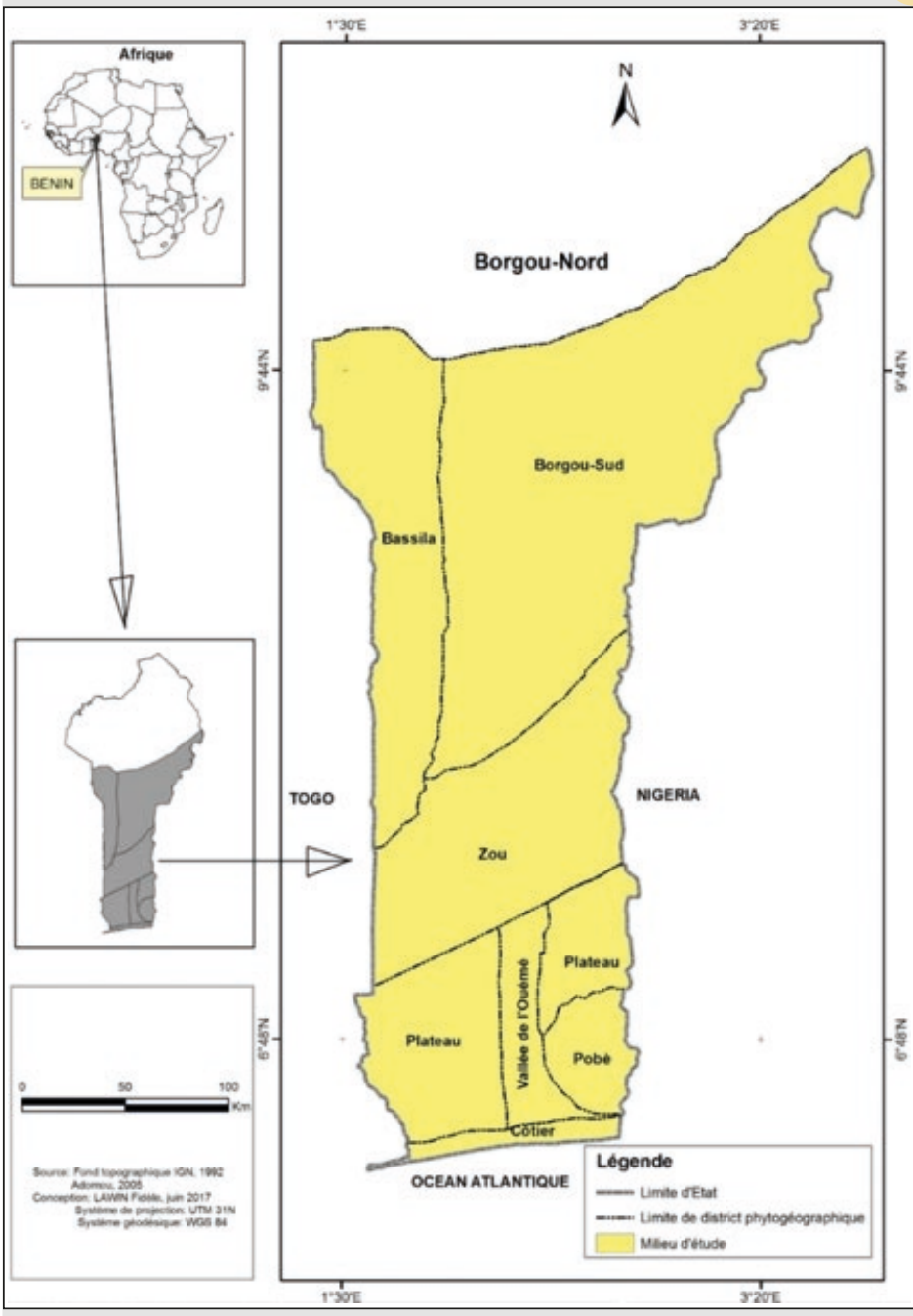

Figure 1.

Positionnement géographique du milieu d'étude.

Dans la zone guinéenne, le régime pluviométrique est bimodal, avec une pluviométrie annuelle de 900 à $1300 \mathrm{~mm}$. L'humidité relative varie entre $69 \%$ et $97 \%$ et la température annuelle moyenne entre $25^{\circ} \mathrm{C}$ et $29{ }^{\circ} \mathrm{C}$ (Adomou, 2005). Les sols sont ferralitiques, profonds et peu fertiles. La végétation primitive est constituée de forêts denses humides semi-décidues et de savanes guinéennes.

Dans la zone soudano-guinéenne, le régime pluviométrique est bimodal à unimodal, et la pluviométrie annuelle moyenne varie entre 1100 et 1200 mm. L'humidité relative varie de $31 \%$ à $98 \%$ et la température annuelle de $25^{\circ} \mathrm{C}$ à $29^{\circ} \mathrm{C}$ (Adomou, 2005). Les sols sont ferrugineux à fertilité variable. La végétation est caractérisée par une mosaïque de forêts claires, de savanes arborées et arbustives, de forêts denses sèches et de forêts-galeries.

Plusieurs groupes socioculturels sont rencontrés dans le milieu d'étude, parmi lesquels les Adja, Sahouè, Kotafon, Fon, Mahi, Aïzo, Wémè, Nagot, Holli, Idaasha, Chabè et Anii. 
Tableau I.

Taille de l'échantillon selon les groupes socioculturels et le genre.

\begin{tabular}{l|c|r|r|}
$\begin{array}{l}\text { Groupes } \\
\text { socioculturels }\end{array}$ & Hommes & $\begin{array}{c}\text { Nombre } \\
\text { Femmes }\end{array}$ & Total \\
\hline Adja & 28 & 23 & 51 \\
\hline Aïzo & 66 & 70 & 136 \\
\hline Anii & 83 & 61 & 144 \\
\hline Chabè & 97 & 29 & 126 \\
\hline Fon & 64 & 51 & 115 \\
\hline Holli & 62 & 34 & 96 \\
\hline Idaasha & 72 & 32 & 104 \\
\hline Kotafon & 24 & 30 & 54 \\
\hline Mahi & 49 & 10 & 59 \\
\hline Nagot & 31 & 31 & 62 \\
\hline Sahouè & 12 & 11 & 23 \\
\hline Wémè & 19 & 20 & 39 \\
\hline Total & 607 & 402 & 1009 \\
\hline
\end{tabular}

Choix des villages d'enquête et échantillonnage

Dans chaque district phytogéographique, les villages à proximité des formations végétales abritant $C$. millenii ont été retenus. Dans chaque village, une enquête exploratoire a été effectuée auprès de 30 personnes choisies aléatoirement. Elle a permis de déterminer la proportion $(P)$ des répondants ayant connaissance d'au moins une utilisation de l'espèce. Cette proportion variait de 0,6 à 0,96. L'effectif n de la population à enquêter a alors été déterminé par la formule de Dagnélie (1998) :
$\mathrm{n}=\frac{\mathrm{U}_{1-\mathrm{a} / 2}^{2} \times \mathrm{P}(1-\mathrm{P})}{d^{2}}$

où $\mathrm{n}$ est la taille de l'échantillon; $\mathrm{P}$ est la proportion de personnes ayant connaissance d'au moins une utilisation de l'espèce lors du sondage $; U_{1-a / 2}=1,96$ en tant que valeur de la variable aléatoire normale pour une valeur de probabilité de $a=0,05$; d est la marge d'erreur fixée à $8 \%$.

La taille de l'échantillon par village variait de 23 à 144, pour un total de 1009 personnes (tableau I) relevant de 12 groupes socioculturels (tableau I) répartis dans 20 villages.

\section{Enquêtes ethnobotaniques}

Chaque personne enquêtée a été soumise à une interview structurée basée sur un questionnaire. Les données collectées concernaient les usages et catégories d'usage de l'espèce, son nom et sa signification en langue locale, les parties commercialisées, ainsi que les menaces et stratégies de conservation de l'espèce.

\section{Traitement des données}

Au sein de la diversité des usages médicinaux renseignés, le choix des parties de l'espèce et leur mode de préparation dans la médecine traditionnelle ont été précisés. La fréquence relative de citation (FRC) et l'indice d'efficacité (IF) ont été utilisés pour apprécier respectivement l'importance relative de chaque utilisation et le niveau d'efficacité de la plante dans le traitement d'une maladie donnée (tableau II).

Dans le but de déterminer la distribution des connaissances dans la population, les personnes interrogées ont été regroupées selon leur appartenance socioculturelle. Six sous-groupes ont été constitués selon l'âge et le genre (tableau III) : les jeunes ont moins de 30 ans, les adultes entre

Tableau II.

Indices ethnobotaniques.

Indices

Fréquence relative de citation (FRC)

Indice d'efficacité (IF)

\section{Formule}

$F R C=\frac{n}{N} \times 100$

$n:$ nombre de personnes ayant fourni une réponse par rapport à une utilisation donnée ; $N$ : nombre total de personnes enquêtées

$I F=\frac{N s}{N t}$

Ns : nombre de personnes ayant obtenu satisfaction après usage d'une partie de C. millenii dans le traitement d'une maladie ; $N t$ : nombre total de personnes ayant utilisé une partie de la plante pour le traitement de la maladie

\section{Interprétation}

Importance relative de chaque utilisation

Niveau d'efficacité d'une plante dans le traitement d'une maladie donnée. On considère que la plante : - n'est pas efficace si IF $<0,25$ - est peu efficace si $0,25 \leq \mathrm{IF}<0,5$ - est moyennement efficace si $0,5 \leq \mathrm{IF}<0,75$ - est très efficace si $0,75 \leq \mathrm{IF} \leq 1$
Références

Tardio et Pardo-DeSantayana (2008)

Présente étude 
Tableau III.

Répartition des enquêtés en fonction des classes d’âge et du genre.

\begin{tabular}{|l|c|c|c|}
\hline Genre & \multicolumn{3}{|c|}{ Âge } \\
& Jeunes & Adultes & Personnes âgées \\
\hline Hommes & 52 & 305 & 250 \\
\hline Femmes & 42 & 202 & 158 \\
\hline
\end{tabular}

30 et 59 ans, et les personnes âgées ont au moins 60 ans (Assogbadjo et al., 2008). Les tableaux de contingence renseignant le nombre de personnes enquêtées utilisant chaque partie de la plante par groupe socioculturel, et par classe d'âge et genre ont été établis. Les usages déclarés par les groupes socioculturels ont été regroupés en catégories d'usage et ont également fait l'objet d'établissement d'un tableau de contingence sur la base du nombre d'utilisateurs par groupe socioculturel. Une analyse factorielle des correspondances (AFC) a été réalisée sur chacun de ces tableaux avec le logiciel $R$ version 3.4.1 afin de décrire non seulement la relation entre les groupes socioculturels et les catégories d'usage mais aussi la relation entre les groupes socioculturels et les parties utilisées, d'une part, et celle entre les classes d'âge et de genre et les parties utilisées, d'autre part.

\section{Résultats}

\section{Noms vernaculaires et signification}

Au total, 17 noms vernaculaires ont été recensés (tableau IV). Ils varient d'un groupe socioculturel à un autre, et parfois d'un village à un autre au sein d'un même groupe socioculturel. L'attribution du nom à la plante repose sur ses traits morphologiques (photo 1) ou sur un évènement historique. C'est le cas par exemple du nom Ola N'gor, signifiant " grâce à cola » en chabè, en allusion à un évènement historique dans la vie de cette communauté : un chasseur affamé en forêt aperçoit $C$. millenii et, après avoir consommé la pulpe, rend grâce à l'espèce pour avoir assouvi sa faim.

\section{Catégories d'usage}

Cola millenii relève de huit catégories d'usage (tableau V). L'usage alimentaire de la pulpe est le plus fréquent (FRC $=96,9 \%)$, suivi de l'usage médicinal $(F R C=66,6 \%)$ et du bois énergie ( $F R C=58,6 \%)$.

\section{Utilisations médicinales}

\section{Maladies et symptômes traités}

C. millenii contribue au traitement de 60 maladies et symptômes (tableau VI). Son utilisation dans le traitement du paludisme est la plus connue de la population (53,9 \%). L'espèce est dite très efficace dans le traitement

Tableau IV.

Noms vernaculaires de Cola millenii et signification.

\begin{tabular}{|c|c|c|c|}
\hline $\begin{array}{l}\text { Groupes } \\
\text { socioculturels }\end{array}$ & $\begin{array}{l}\text { Noms vernaculaires } \\
\text { vernaculaires }\end{array}$ & Significations & Villages \\
\hline Anii & Abatankouyabou, Abatankouporo & - & Baka baka (Bassila) \\
\hline Chabè & Ola N'gor & Grâce à cola & Koda (Tchaourou) \\
\hline Kotafon & Pafoli & - & Gbédji (Houéyogbé) \\
\hline Fon & $\begin{array}{l}\text { Alovi aton } \\
\text { Zinkokoé, Zinwokokoé } \\
\text { Kèklègba }\end{array}$ & $\begin{array}{l}\text { Cinq doigts } \\
\text { Banane du singe }\end{array}$ & $\begin{array}{l}\text { Niaouli } 1 \text { (Allada) } \\
\text { Attakpacodji (Ouidah) } \\
\text { Niaouli } 1 \\
\text { Lokoli (Zogbodomè) }\end{array}$ \\
\hline Aïzo & Alovi aton & Cinq doigts & Ayou (Allada) \\
\hline Wémè & Alovi aton & Cinq doigts & Tovoh (Bonou) \\
\hline Idaasha & Bototo & - & Modji-gangan, (Dassa) \\
\hline Mahi & $\begin{array}{l}\text { Katanangba, } \\
\text { Lègba-kèkè }\end{array}$ & - & Adakplamè (Kétou) \\
\hline Nagot & Kaka N’gba & - & Ahoyéyé (Pobè) \\
\hline Holli & Kakara N'gba & - & Issaba (Pobè) \\
\hline Adja & $\begin{array}{l}\text { Lovi aton, } \\
\text { Gbambo }\end{array}$ & Cinq doigts & Yéhouémey (Aplahoué) \\
\hline Sahouè & $\begin{array}{l}\text { Didonvè } \\
\text { Gbankpo }\end{array}$ & - & $\begin{array}{l}\text { Zoungbonou (Houéyogbé) } \\
\text { Sè (Houéyogbé) }\end{array}$ \\
\hline
\end{tabular}


Tableau V.

Catégories d'usage de Cola millenii.

\section{Catégories d'usage}

Médicinale

Alimentaire

Brosse végétale

Emballage

Magique

\section{Parties utilisées}

Feuille, écorce, racine, Traitement des maladies graine, pulpe et fruit

Pulpe

Racine

Feuille

Feuille

Feuille

Feuille

Bois de service

Bois énergie

Bois d'œuvre

\section{Tronc, branche}

Tronc
Observations

Consommée crue pour attirer la chance

Gaules, pieux
FRC (\%)

66,6

96,9

Utilisée comme cure-dents

2,0

Utilisée pour emballer l'akassa

0,7

Feuille attachée + incantation pour arrêter un voleur

0,4

Feuilles de C. millenii + autres feuilles gardées sur soi

Feuille en décoction + autres plantes utilisées en bain corporel une fois par an pour se prémunir des mauvais esprits

Bois de feu et charbon de bois

Bois de charpente, fabrication de tabourets

Tableau VI.

Remèdes médicinaux à base de Cola millenii.

\begin{tabular}{|c|c|c|c|c|c|c|c|c|}
\hline $\begin{array}{l}\text { Catégories } \\
\text { d'affections }\end{array}$ & $\begin{array}{l}\text { Maladies } \\
\text { et symptômes }\end{array}$ & $\begin{array}{l}\text { Partie } \\
\text { utilisée }\end{array}$ & $\begin{array}{l}\text { Mode de } \\
\text { préparation }\end{array}$ & $\begin{array}{l}\text { Mode } \\
\text { d'administration }\end{array}$ & $\begin{array}{l}\text { Groupes } \\
\text { socioculturels }\end{array}$ & Villages & $\begin{array}{l}\text { FRC } \\
(\%)\end{array}$ & IF \\
\hline Hématologique & Anémie & Feuille & Décoction & Boisson & Holli, Idaasha & Issaba, Modji-gangan & 0,20 & 0,50 \\
\hline Endocrinienne & Diabète & Feuille & Décoction & Boisson & Aïzo, Idaasha & Niaouli 1, Kpingni & 0,20 & 0,50 \\
\hline Génétique & Drépanocytose & Racine & Décoction & Bain corporel & Aïzo & Ayou & 0,20 & 0,50 \\
\hline \multirow[t]{6}{*}{ Traumatiques } & \multirow[t]{2}{*}{ Hernie } & Fruit & Poudre & Boisson & \multirow[t]{2}{*}{ Fon, Holli } & \multirow[t]{2}{*}{ Attakpacodji, Issaba } & \multirow[t]{2}{*}{0,34} & \multirow[t]{2}{*}{0,33} \\
\hline & & $\begin{array}{l}\text { Graine } \\
\text { et racine }\end{array}$ & Macération & Boisson & & & & \\
\hline & Hémorroïde & Écorce & Macération & Boisson & $\begin{array}{l}\text { Adja, Aïzo, } \\
\text { Fon, Holli }\end{array}$ & $\begin{array}{l}\text { Yéhouémey, Ayou, Attakpacodji, } \\
\text { Issaba }\end{array}$ & 0,40 & 0,62 \\
\hline & Entorse & Feuille & Décoction & Application locale & Chabè & Koda & 0,10 & - \\
\hline & Plaie & Écorce & Poudre & Application locale & Chabè, Fon & Koda, Attakpacodji & 0,20 & 0,50 \\
\hline & Abcès & Feuille & Décoction & Boisson & Kotafon & Gbédji & 0,30 & 0,33 \\
\hline \multirow[t]{9}{*}{ Gynécologiques } & Reflux de sperme & Écorce & Macération & Boisson & Fon & Niaouli 1 & 0,20 & 0,50 \\
\hline & Avortement précoce & Écorce & Macération & Boisson & Aïzo & Niaouli 1 & 0,10 & - \\
\hline & Oligospermie & $\begin{array}{l}\text { Écorce } \\
\text { ou racine }\end{array}$ & Macération & Boisson & $\begin{array}{l}\text { Aïzo, Fon, } \\
\text { Idaasha, Wémè }\end{array}$ & $\begin{array}{l}\text { Ayou, Niaouli 1, Modji-gangan, } \\
\text { Tovoh }\end{array}$ & 5,08 & 0,25 \\
\hline & $\begin{array}{l}\text { Saignements chez } \\
\text { la femme enceinte }\end{array}$ & Feuille & Trituration & Boisson & Aïzo, Fon & Ayou, Niaouli 1 & 0,20 & - \\
\hline & $\begin{array}{l}\text { Stérilité féminine } \\
\text { ou masculine }\end{array}$ & Graine & Poudre & $\begin{array}{l}\text { Mélange } \\
\text { alimentaire }\end{array}$ & $\begin{array}{l}\text { Adja, Aïzo, Fon, } \\
\text { Wémè, Mahi, } \\
\text { Sahouè }\end{array}$ & $\begin{array}{l}\text { Yéhouémey, Ayou, attakpacodji, } \\
\text { Tovoh, Kpingni, Zoungbonou }\end{array}$ & 0,59 & 0,25 \\
\hline & Ménopause précoce & $\begin{array}{l}\text { Écorce } \\
\text { et feuille }\end{array}$ & Décoction & Bain corporel & Idaasha & Modji-gangan & 0,20 & - \\
\hline & \multirow[t]{2}{*}{ Règles douloureuses } & Feuille & Décoction & Boisson & \multirow[t]{2}{*}{ Chabè, Wémè } & \multirow[t]{2}{*}{ Koda, Tovoh } & \multirow[t]{2}{*}{1,00} & \multirow[t]{2}{*}{-} \\
\hline & & Racine & Décoction & Boisson & & & & \\
\hline & Règles irrégulières & Racine & Décoction & Boisson & Idaasha, Nagot & Modji-gangan, Ahoyéyé & 0,20 & - \\
\hline
\end{tabular}


Tableau VI (suite).

\begin{tabular}{|c|c|c|c|c|c|c|c|c|}
\hline $\begin{array}{l}\text { Catégories } \\
\text { d'affections }\end{array}$ & $\begin{array}{l}\text { Maladies } \\
\text { et symptômes }\end{array}$ & $\begin{array}{l}\text { Partie } \\
\text { utilisée }\end{array}$ & $\begin{array}{l}\text { Mode de } \\
\text { préparation }\end{array}$ & $\begin{array}{l}\text { Mode } \\
\text { d'administration }\end{array}$ & $\begin{array}{l}\text { Groupes } \\
\text { socioculturels }\end{array}$ & Villages & $\begin{array}{l}\text { FRC } \\
(\%)\end{array}$ & IF \\
\hline Gynécologiques & $\begin{array}{l}\text { Troubles liés } \\
\text { à la grossesse }\end{array}$ & Feuille & Décoction & Boisson & $\begin{array}{l}\text { Adja, Aïzo, } \\
\text { Fon, Kotafon }\end{array}$ & $\begin{array}{l}\text { Yéhouémey, Kpotomey, } \\
\text { Dohouignantomè, Niaouli 1, Gbédji }\end{array}$ & 6,55 & 0,52 \\
\hline \multirow[t]{17}{*}{ Digestives } & Constipation & Feuille & Décoction & Boisson & Aïzo, Idaasha & Niaouli 1, Modji-gangan & 0,49 & 0,50 \\
\hline & \multirow[t]{2}{*}{ Perte d’appétit } & Feuille & Décoction & Boisson & \multirow[t]{2}{*}{ Kotafon } & \multirow[t]{2}{*}{ Gbédji } & \multirow[t]{2}{*}{0,68} & \multirow[t]{2}{*}{0,65} \\
\hline & & Racine & - & Comme cure-dents & & & & \\
\hline & Vomissements & Feuille & Décoction & Boisson & Aïzo, Fon & Ayou, Attakpacodji & 0,20 & - \\
\hline & Diarrhée & Feuille & Décoction & Boisson & Aïzo, Fon & Ayou, Attakpacodji & 0,20 & - \\
\hline & Dysenterie & Feuille & Décoction & Boisson & Aïzo, Chabè & Ayou, Koda & 0,20 & 0,50 \\
\hline & & Écorce & Poudre & Mélange alimentaire & & & & \\
\hline & \multirow[t]{3}{*}{ Maux de ventre } & Graine & Macération & Boisson & \multirow[t]{3}{*}{$\begin{array}{l}\text { Adja, Chabè, Fon, } \\
\text { Kotafon, Wémè }\end{array}$} & \multirow[t]{3}{*}{$\begin{array}{l}\text { Yéhouémey, Koda, Attakpacodji, } \\
\text { Gbédji, Tovoh }\end{array}$} & \multirow[t]{3}{*}{6,33} & \multirow[t]{3}{*}{0,86} \\
\hline & & $\begin{array}{l}\text { Écorce } \\
\text { fraîche }\end{array}$ & Macération & Boisson & & & & \\
\hline & & Racine & Macération & Boisson & & & & \\
\hline & $\begin{array}{l}\text { Infection buccale } \\
\text { (plaie dans la bouche) }\end{array}$ & Pulpe & - & Succion & Wémè & Tovoh & 0,20 & - \\
\hline & Mauvaise haleine & Racine & - & Comme cure-dents & Sahouè & Zoungbonou & 0,20 & - \\
\hline & \multirow[t]{3}{*}{ Ulcère } & Feuille & Décoction & Boisson & \multirow[t]{3}{*}{$\begin{array}{l}\text { Adja, Aïzo, Fon, } \\
\text { Holli, Wémè, } \\
\text { Mahi, Sahouè }\end{array}$} & \multirow[t]{3}{*}{$\begin{array}{l}\text { Yéhouémey, Ayou, Attakpacodji, } \\
\text { Niaouli 1, Issaba, Tovoh, Adakplamè, } \\
\text { Zoungbonou }\end{array}$} & \multirow[t]{3}{*}{0,79} & \multirow[t]{3}{*}{0,57} \\
\hline & & Racine & Macération & Boisson & & & & \\
\hline & & Écorce & Macération & Boisson & & & & \\
\hline & Infection abdominale & Feuille & Décoction & Boisson & Fon & Attakpacodji & 0,30 & - \\
\hline & Nausée & Feuille & Décoction & Boisson & Adja & Yéhouémey & 0,20 & - \\
\hline \multirow[t]{5}{*}{ Dermatologiques } & Gale & Écorce & Macération & Bain corporel & Idaasha & Modji-gangan & 0,20 & - \\
\hline & Teigne & Écorce & Décoction & Bain corporel & Chabè & Koda & 0,20 & - \\
\hline & Syphilis & Feuille & Trituration & Boisson & $\begin{array}{l}\text { Aïzo, Fon, } \\
\text { Idassha }\end{array}$ & $\begin{array}{l}\text { Ayou, Dohouignantomè, } \\
\text { Modji-gangan }\end{array}$ & 0,30 & - \\
\hline & Variole & Feuille & Décoction & Bain corporel & Chabè & Koda & 0,20 & - \\
\hline & Rougeole & Feuille & Trituration & Bain corporel & Chabè & Koda & 0,30 & - \\
\hline Psychologiques & Éjaculation précoce & Racine & Macération & Boisson & Idaasha & Modji-gangan & 0,20 & - \\
\hline \multirow[t]{2}{*}{ Cardiologiques } & Hypertension & $\begin{array}{l}\text { Feuille } \\
\text { ou écorce }\end{array}$ & Décoction & Boisson & $\begin{array}{l}\text { Adja, Fon, Holli, } \\
\text { Kotafon, Sahouè }\end{array}$ & $\begin{array}{l}\text { Yéhouémey, Attakpacodji, Ayou, } \\
\text { Dohouignantomè, Lokoli, Niaouli 1, } \\
\text { Issaba, Gbédji, Zoungbonou }\end{array}$ & 6,21 & 0,86 \\
\hline & Hypotension & Feuille & Décoction & Boisson & Holli & Issaba & 0,20 & - \\
\hline \multirow[t]{2}{*}{ Stomatologiques } & Carie dentaire & Pulpe & - & Application locale & \multirow[t]{2}{*}{$\begin{array}{l}\text { Adja, Aïzo, Fon, } \\
\text { Kotafon, Mahi, } \\
\text { Sahouè }\end{array}$} & \multirow[t]{2}{*}{$\begin{array}{l}\text { Yéhouémey, Ayou, Niaouli 1, } \\
\text { Attakpacodji, Gbédji, Kpingni, } \\
\text { Zoungbonou }\end{array}$} & \multirow[t]{2}{*}{1,5} & \multirow[t]{2}{*}{0,62} \\
\hline & & Racine & - & Comme cure-dents & & & & \\
\hline \multirow[t]{6}{*}{ Neurologiques } & Maux de tête & Écorce & Macération & Bain de la tête & $\begin{array}{l}\text { Aïzo, Fon, } \\
\text { Kotafon }\end{array}$ & Ayou, Attakpacodji, Gbédji & 0,20 & 0,50 \\
\hline & \multirow[t]{3}{*}{ Crise convulsive } & \multirow[t]{2}{*}{ Feuille } & Décoction & Bain par vapeur & $\begin{array}{l}\text { Aïzo, Holli, } \\
\text { Kotafon }\end{array}$ & Ayou, Niaouli 1, Issaba, Gbédji & 0,56 & 0,40 \\
\hline & & & & Boisson & & & & \\
\hline & & Écorce & Décoction & Bain corporel & & & & \\
\hline & Épilepsie & Racine & Poudre & Lapement & Mahi & Adakplamè & 0,20 & - \\
\hline & Paralysie & Racine & Décoction & Bain corporel & Mahi & Adakplamè, Kpingni & 0,60 & 0,40 \\
\hline Pédiatriques & $\begin{array}{l}\text { Non-solidité du corps } \\
\text { chez le nouveau-né }\end{array}$ & Racine & Décoction & Bain corporel & Sahouè & Zoungbonou & 0,20 & - \\
\hline & Marasme & Feuille & Décoction & Boisson & Chabè & Koda & 0,10 & - \\
\hline
\end{tabular}


Tableau VI (suite).

\begin{tabular}{|c|c|c|c|c|c|c|c|c|}
\hline $\begin{array}{l}\text { Catégories } \\
\text { d'affections }\end{array}$ & $\begin{array}{l}\text { Maladies } \\
\text { et symptômes }\end{array}$ & $\begin{array}{l}\text { Partie } \\
\text { utilisée }\end{array}$ & $\begin{array}{l}\text { Mode de } \\
\text { préparation }\end{array}$ & $\begin{array}{l}\text { Mode } \\
\text { d'administration }\end{array}$ & $\begin{array}{l}\text { Groupes } \\
\text { socioculturels }\end{array}$ & Villages & $\begin{array}{l}\text { FRC } \\
(\%)\end{array}$ & IF \\
\hline \multirow[t]{2}{*}{ Pédiatriques } & $\begin{array}{l}\text { Dentition difficile } \\
\text { chez l'enfant }\end{array}$ & Pulpe & Macération & Boisson & Holli, Sahouè & Issaba, Zoungbonou & 0,20 & - \\
\hline & $\begin{array}{l}\text { Retard de l'enfant } \\
\text { à marcher }\end{array}$ & Feuille & Décoction & Bain corporel & Holli & Issaba & 0,10 & - \\
\hline \multirow[t]{19}{*}{ Autres } & Fièvre & $\begin{array}{l}\text { Ecorce } \\
\text { ou feuille }\end{array}$ & Décoction & Boisson & $\begin{array}{l}\text { Adja, Aïzo, Fon, } \\
\text { Holli, Idaasha, } \\
\text { Kotafon, Sahouè }\end{array}$ & $\begin{array}{l}\text { Yéhouémey, Attakpacodji, Ayou, } \\
\text { Lokoli, Niaouli 1, Issaba, } \\
\text { Modji-gangan, Gbédji, Zoungbonou }\end{array}$ & 5,54 & 0,78 \\
\hline & Vertige & Feuille & Décoction & Boisson & \multirow[t]{2}{*}{$\begin{array}{l}\text { Aïzo, Chabè, } \\
\text { Fon, Idaasha }\end{array}$} & \multirow[t]{2}{*}{$\begin{array}{l}\text { Ayou, Koda, Attakpacodji, } \\
\text { Dohouignantomè, Modji-gangan }\end{array}$} & \multirow[t]{2}{*}{5,20} & \multirow[t]{2}{*}{0,80} \\
\hline & & Écorce & Décoction & Bain corporel & & & & \\
\hline & Courbatures & Feuille & Décoction & Boisson & $\begin{array}{l}\text { Adja, Aïzo, } \\
\text { Chabè, Fon, } \\
\text { Kotafon, } \\
\text { Idaasha, Sahouè }\end{array}$ & $\begin{array}{l}\text { Yéhouémey, Ayou, Koda, } \\
\text { Attakpacodji, Niaouli 1, Gbédji, } \\
\text { Modji-gangan, Zoungbonou }\end{array}$ & 5,99 & 0,78 \\
\hline & Fatigue & Feuille & Décoction & Boisson & $\begin{array}{l}\text { Adja, Kotafon, } \\
\text { wémè }\end{array}$ & Yéhouémey, Gbédji, Tovoh & 5,87 & 0,87 \\
\hline & Toux & Feuille & Décoction & Boisson & $\begin{array}{l}\text { Aïzo, Chabè, } \\
\text { Fon, Idaasha }\end{array}$ & $\begin{array}{l}\text { Ayou, Koda, Attakpacodji, } \\
\text { Modji-gangan }\end{array}$ & 0,40 & 0,50 \\
\hline & Enflure des pieds & Écorce & Poudre & Application locale & $\begin{array}{l}\text { Adja, Chabè, } \\
\text { Fon, Kotafon, } \\
\text { Mahi, Sahouè }\end{array}$ & $\begin{array}{l}\text { Yéhouémey, Koda, Attakpacodji, } \\
\text { Ayou, Dohouignantomè, Lokoli, } \\
\text { Niaouli 1, Gbédji, Kpingni, } \\
\text { Modji-gangan, Zoungbonou }\end{array}$ & 5,65 & 0,60 \\
\hline & & Feuille & Décoction & Boisson & & & & \\
\hline & Rhume & Feuille & Décoction & Boisson & Chabè & Koda & 0,20 & - \\
\hline & Asthme & Feuille & Brûlage & Inhalation & $\begin{array}{l}\text { Adja, Fon, } \\
\text { Kotafon }\end{array}$ & $\begin{array}{l}\text { Attakpacodji, Yéhouémey, } \\
\text { Dohouignantomè, Gbédji }\end{array}$ & 6,55 & 0,66 \\
\hline & Angine & Feuille & Décoction & Boisson & Fon & Attakpacodji, Niaouli 1 & 0,20 & - \\
\hline & & Racine & Macération & Lavage de gorge & & & & \\
\hline & Enflure du corps & Feuille & Décoction & Bain corporel & Adja, Idaasha & Attakpacodji, Modji-gangan & 0,20 & - \\
\hline & Douleur lombaire & Racine & Décoction & Boisson & Kotafon, Wémè & Gbédji Tovoh, & 0,20 & 0,50 \\
\hline & Faiblesse sexuelle & Écorce & Macération & Boisson & Wémè & Tovoh & 0,20 & - \\
\hline & Paludisme & $\begin{array}{l}\text { Feuille verte } \\
\text { (ou feuille } \\
\text { jaune } \\
\text { tombée par } \\
\text { terre) }\end{array}$ & Décoction & Boisson & $\begin{array}{l}\text { Adja, Aïzo, Chabè, } \\
\text { Fon, Holli, } \\
\text { Idaasha, Kotafon, } \\
\text { Nago, Wémè, } \\
\text { Mahi, Sahouè }\end{array}$ & $\begin{array}{l}\text { Yéhouémey, Kpotomey, Lanzron, } \\
\text { Zinvié, Koda, Attakpacodji, Ayou, } \\
\text { Dohouignantomè, Lokoli, Niaouli 1, } \\
\text { Issaba, Gbédji, Ahoyéyé, } \\
\text { Hounmè Tovoh, Adakplamè, Kpingni, } \\
\text { Modji-gangan, Zoungbonou }\end{array}$ & 53,90 & 1 \\
\hline & Talon fendillé & Écorce & Décoction & Application locale & Mahi & Adakplamè, Kpingni & 0,20 & - \\
\hline & Ictère & Feuille & Décoction & Boisson & $\begin{array}{l}\text { Aïzo, Chabè, } \\
\text { Fon, Idaasha, } \\
\text { Mahi, Sahouè }\end{array}$ & $\begin{array}{l}\text { Kpotomey, Lanzron, Niaouli 1, } \\
\text { Zinvié, Koda, Attakpacodji, Ayou, } \\
\text { Dohouignantomè, Lokoli, Kpingni, } \\
\text { Modji-gangan, Adakplamè, }\end{array}$ & 7,91 & 0,89 \\
\hline & & Racine & Décoction & Boisson & & Zoungbonou & & \\
\hline
\end{tabular}



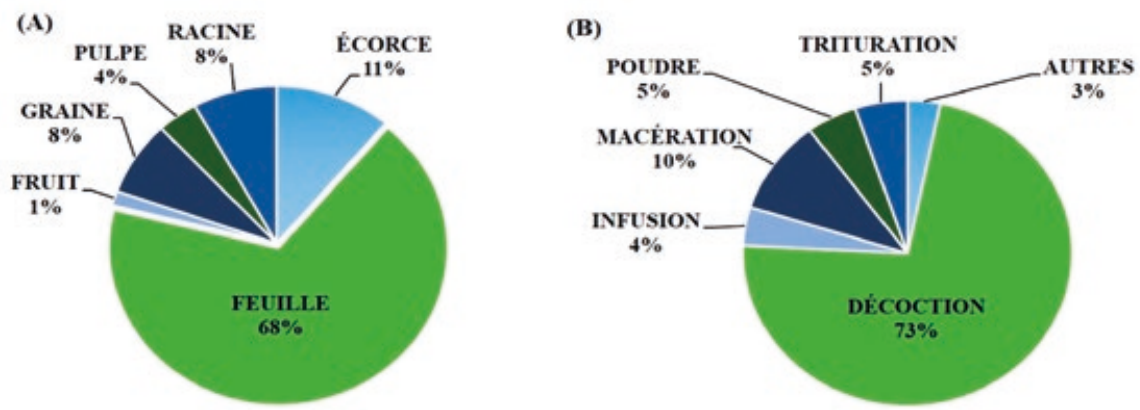

Figure 2.

Parties de Cola millenii utilisées (A); modes de préparation des remèdes (B).

de multiples affections : paludisme, ictère, maux de ventre, hypertension, courbatures, fatigue, fièvre et vertige, mais moyennement efficace dans le traitement de l'asthme, des troubles liés à la grossesse et à l'enflure des pieds, et peu efficace contre l'oligospermie.

Pour renforcer l'efficacité des remèdes mentionnés, d'autres plantes ou minéraux sont parfois associés.

\section{Parties utilisées et modes de préparation}

Les feuilles, les fruits, les graines, les écorces, les racines et la pulpe sont les parties de $C$. millenii utilisées. La feuille est la plus utilisée (68\%) et la décoction est le mode de préparation le plus employé (73\%) (figure 2).

\section{Relation entre groupes socioculturels et catégories d'usage}

Les deux premiers facteurs de l'AFC regroupent $60,7 \%$ de la variance totale. Toutefois, le troisième facteur a également été retenu pour décrire les relations, les trois premiers facteurs de cette AFC totalisant $76,6 \%$ de la variabilité initiale. L'analyse des contributions ainsi que de la qualité de la représentation des groupes socioculturels et des catégories d'usage de $C$. millenii sur chaque axe du plan factoriel principal indique que les groupes socioculturels Anii, Chabè, Adja et Kotafon présentent une bonne contribution et une bonne qualité de représentation sur l'axe factoriel 1, alors que les Aïzo et les Mahi révélaient respectivement de bonnes contributions et qualités de représentation sur les axes factoriels 2 et 3 . Les catégories d'usage alimentaire et magique et, dans une certaine mesure, la valorisation sous forme de brosse végétale et de bois de service contribuent fortement à la formation de l'axe 1 et y sont bien représentées, alors que les catégories relatives à l'usage médicinal et au bois d'œuvre présentent respectivement de bonnes contributions et qualités de représentation sur les axes factoriels 2 et 3 . Les correspondances issues de la projection des différentes catégories d'usage et des groupes socioculturels dans les systèmes d'axes de l'AFC (figure 3) montrent que les Anii et Chabè préfèrent l'usage alimentaire de l'espèce, tandis que son usage à des fins magiques et comme brosse végétale (cure-dents) et bois de service est privilégié par les Adja et Kotafon. Par contre, les Aïzo l'utilisent plutôt à des fins médicinales et son usage comme bois d'œuvre est plus spécifique des Mahi. Les autres groupes socioculturels, non encerclés sur la figure 3 , ne sont pas associés à une catégorie d'usage particulière.

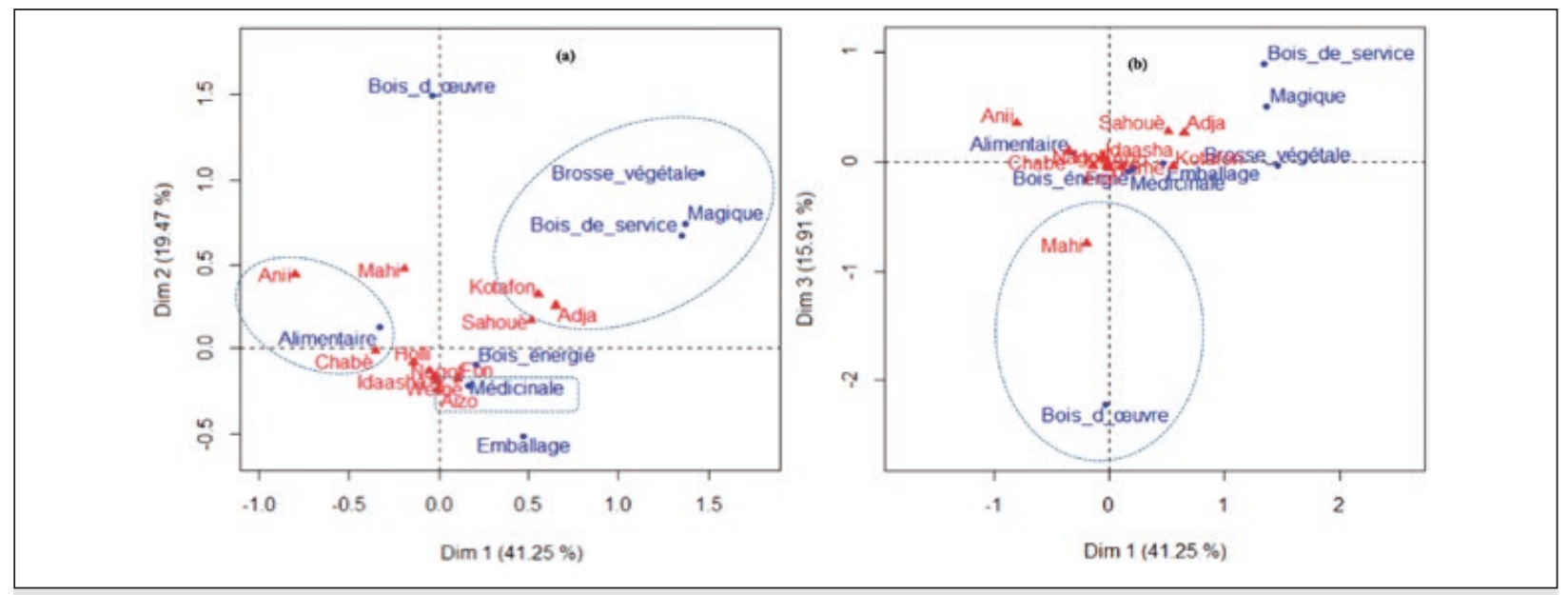

Figure 3.

Projection des catégories d'usage de Cola millenii et des groupes socioculturels sur les plans factoriels (a) 1 et 2 et (b) 1 et 3. 

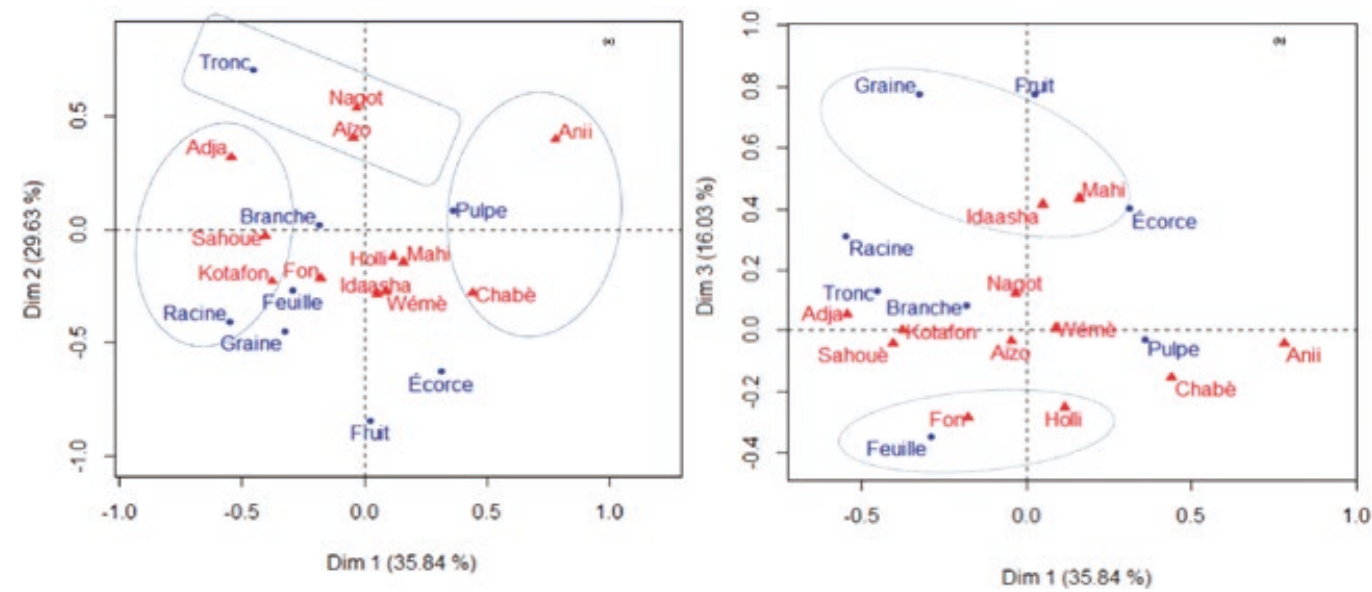

Figure 4.

Projection des parties utilisées de Cola millenii et des groupes socioculturels sur les plans factoriels principaux (a) 1 et 2 et (b) 1 et 3.

\section{Relation entre groupes socioculturels et parties utilisées}

Les résultats de l'analyse indiquent que $65,5 \%$ de la variabilité est portée par les deux premiers axes factoriels. Néanmoins, dans le but de faire ressortir davantage les liens entre les groupes socioculturels et les parties utilisées, le troisième axe factoriel a été aussi pris en compte, et totalise avec les deux premiers axes $81,5 \%$ de la variabilité initiale. L'analyse des contributions ainsi que de la qualité de représentation des groupes socioculturels et parties de $C$. millenii utilisées sur chaque axe factoriel indique que les groupes socioculturels Adja, Anii, Chabè, Kotafon et Sahouè avaient une bonne contribution et une bonne qualité de représentation sur l'axe factoriel 1 alors que les Aïzo et Nagot, d'une part, et les Fon, Holli, Idaasha et Mahi, d'autre part, avaient une bonne contribution et une bonne qualité de représentation sur l'axe factoriel 2 et l'axe factoriel 3, respectivement. S'agissant des parties utilisées, la pulpe et la racine présentaient une bonne contribution et une bonne qualité de représentation sur

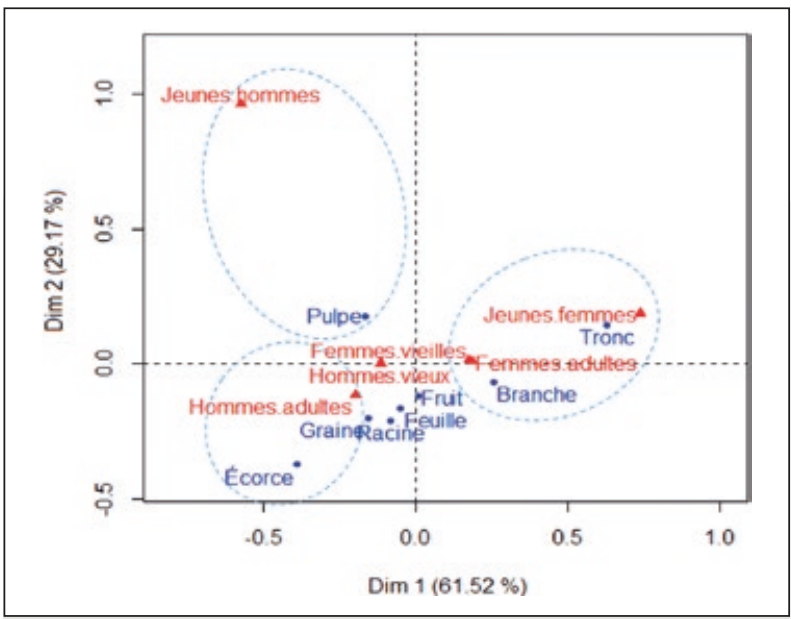

Figure 5.

Projection des utilisations des parties de Cola millenii et des classes d'âge et de genre sur le plan factoriel principal de l'AFC. l'axe 1, alors que le tronc et les écorces, d'une part, et la graine et la feuille, d'autre part, avaient une bonne contribution et une bonne qualité de représentation sur l'axe 2 et l'axe 3, respectivement. Les correspondances issues de la projection des différentes parties utilisées et des groupes socioculturels dans les systèmes d'axes de l'AFC (figure 4) montrent que les Adja, Kotafon et Sahouè accordent plus d'importance à la racine alors que les Anii et Chabè se reportent davantage sur l'utilisation de la pulpe. Les Aïzo et Nagot s'intéressent plus à l'utilisation du tronc. Les Fon et Holli préfèrent l'usage des feuilles tandis que les Idaasha et Mahi privilégient l'utilisation de la graine. Les autres groupes socioculturels ne sont pas associés à l'utilisation particulière d'une partie de la plante.

\section{Relation entre âge-genre et parties utilisées}

Les résultats de l'AFC, dont les deux premiers facteurs regroupent $90,7 \%$ des informations, indiquent que les femmes, jeunes, adultes ou vieilles, préfèrent l'utilisation du tronc et de la branche par rapport aux autres sous-groupes (figure 5). Ces usages s'opposent à celui des écorces chez les hommes adultes. Quant aux jeunes hommes, ils utilisent plutôt la pulpe. Les autres connaissances sur l'utilisation des parties de $C$. millenii ne discriminent pas les classes d'âge et le genre parce qu'elles ne sont pas significativement exprimées sur les axes factoriels.

\section{Commercialisation}

Dans la zone d'étude, C. millenii fait l'objet d'une commercialisation locale des feuilles et fruits, mais aussi des racines, écorces et graines (figure 6). Les feuilles et les fruits sont les plus commercialisés, respectivement dans les zones guinéenne et soudano-guinéenne.

Les fruits sont vendus à 5 FCFA l'unité ou 25 FCFA le tas de six fruits aux abords des voies (photo 2), dans les quartiers urbains, dans certaines écoles primaires et dans les marchés (photo 3), au sud et au centre du Bénin. Les clients sont surtout des enfants. Les feuilles sont commercialisées en bottes (photo 4) de 25 à 100 FCFA selon la taille de la 


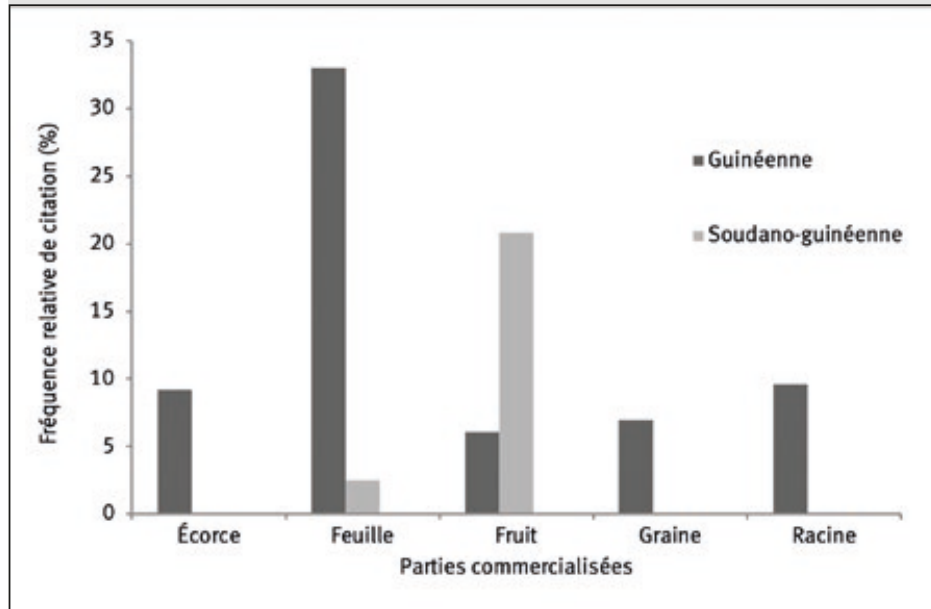

Figure 6.

Parties de Cola millenii commercialisées dans les deux zones étudiées.

botte, dans plusieurs marchés au sud du Bénin, principalement à Abomey-Calavi et Dantokpa. Les graines, écorces et racines sont vendues en tas de 25 FCFA ou 50 FCFA, selon la grandeur du tas, dans certains marchés du Sud-Bénin, mais de manière occasionnelle.

\section{Menaces et stratégies de conservation}

C. millenii subit diverses pressions. La coupe pour le prélèvement de bois de chauffe, le défrichement de son habitat à des fins agricoles, les feux de végétation et l'urbanisation sont les principales menaces en jeu (figure 7).

Seule l'épargne des pieds au champ et dans les agglomérations a été identifiée comme stratégie de conservation, par seulement $3 \%$ des personnes enquêtées.

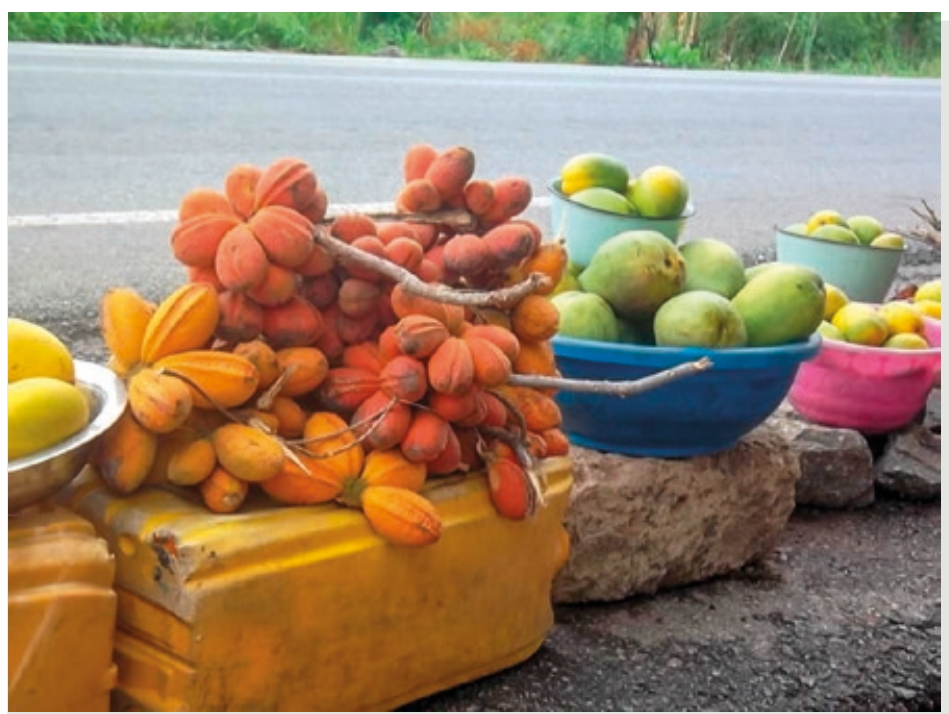

Photo 2.

Fruits de Cola millenii vendus au bord de la voie, à côté des mangues à Modji-gangan (Dassa). Photo I. F. Lawin.

\section{Discussion}

\section{Noms vernaculaires}

Les noms varient d'un groupe socioculturel à l'autre, parfois d'un village à l'autre pour un même groupe socioculturel. L'attribution du nom à la plante repose non seulement sur ses traits morphologiques mais aussi sur un événement historique (Achigan-Dako et al., 2010 ; Houètchégnon et al., 2015 ; Assogba et al., 2017). Cette connaissance suppose l'existence d'une longue histoire de l'utilisation de l'espèce par les communautés concernées (Houètchégnon et al., 2015 ; Assogba et al., 2017). Le nom vernaculaire aurait également un lien avec l'usage préférentiel de l'espèce pour chaque groupe socioculturel conformément aux travaux de Assogba et al. (2017). À titre d'exemple, les Chabè désignent $C$. millenii sous le nom Ola N'gor signifiant « grâce à cola », en relation avec l'importance de la consommation de la pulpe du fruit pour ce groupe socioculturel. L'espèce est également appelée alovi aton par les Fon, ce qui signifie « cinq doigts », en référence à la forme pentalobée de la feuille, partie la plus utilisée par ce groupe.

\section{Diversité des usages}

C. millenii relève de plusieurs catégories d'usage. L'usage alimentaire de la pulpe (FRC $=96,9 \%)$ et des parties de l'espèce dans la médecine traditionnelle (FRC $=66,6 \%)$ et comme bois énergie ( $F R C=58,6 \%$ ) sont les plus pratiqués par les personnes enquêtées. Ceci traduit l'importance alimentaire et médicinale de l'espèce pour la population. Elle estime que le bois de $C$. millenii s'enflamme rapidement et ne dure pas dans les foyers, et est donc plutôt considéré comme de mauvaise qualité.

L'espèce est utilisée dans le traitement de 60 maladies et symptômes. Ce nombre est largement supérieur aux 15 maladies et symptômes rapportés dans la littérature

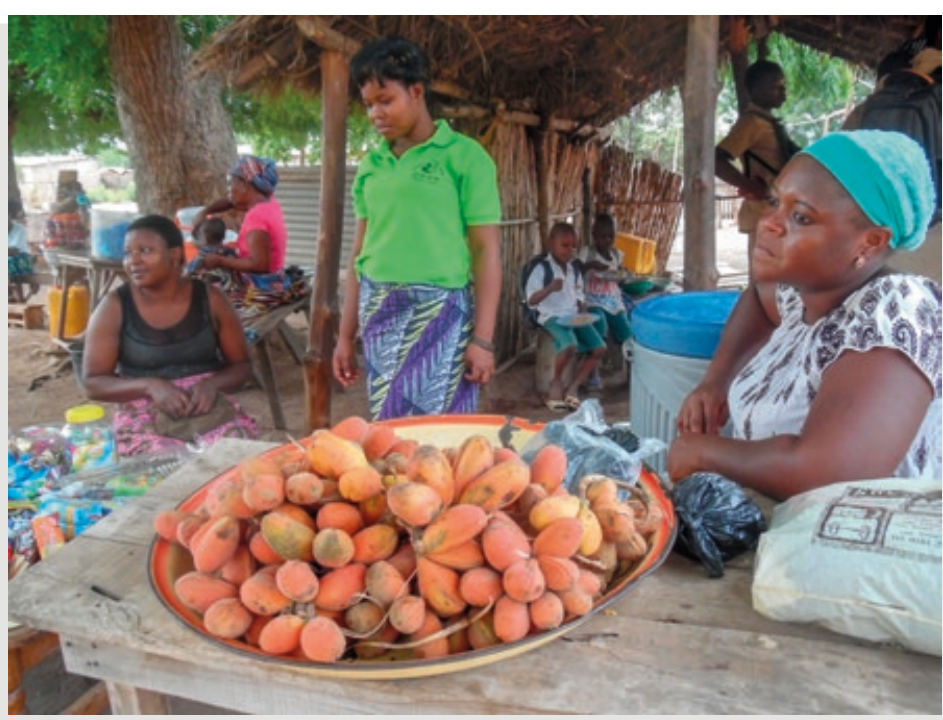

Photo 3.

Fruits de Cola millenii vendus au marché de Dassa. Photo I. F. Lawin. 
Bois et Forêts des Tropiques - ISSN: L-0006-579X

Volume $339-1^{\text {st }}$ quarter - January 2019 - p. 61-74

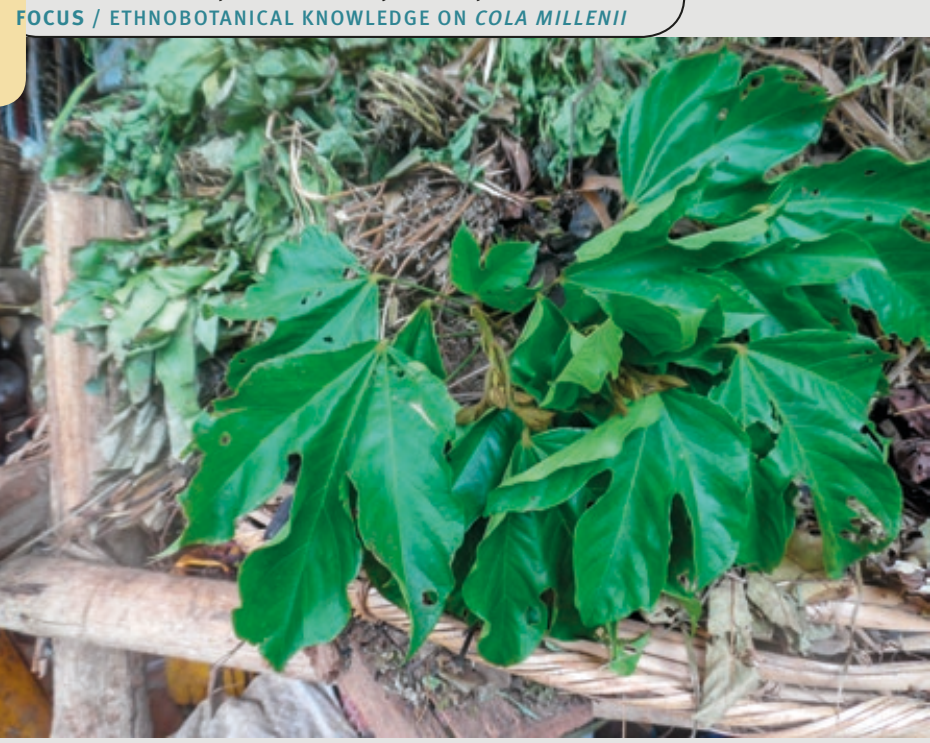

Photo 4.

Feuilles de Cola millenii sur un étalage de plantes médicinales au marché de Dantokpa.

Photo I. F. Lawin.

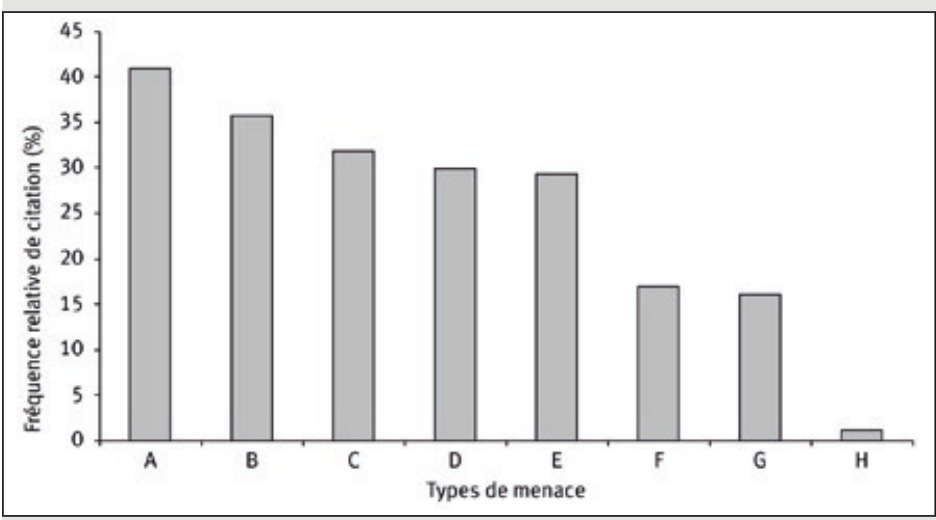

Figure 7.

Menaces pesant sur Cola millenii : A : coupe pour le prélèvement de bois de chauffe ; $B$ : défrichement à des fins agricoles ; $C$ : feux de végétation ; $D$ : urbanisation ; $E$ : prélèvement des parties de la plante à des fins médicinales ; $\mathrm{F}$ : piétinement des plantules et du sol par les bœufs ; $\mathrm{G}$ : fabrication de charbon de bois ;

$\mathrm{H}$ : changement climatique.

(Akoègninou et al., 2006 ; Odugbemi, 2006 ; Adomou et al., 2012 ; Koudouvo et al., 2016 ; Houmenou et al., 2017). Ceci peut s'expliquer par la grande taille de l'échantillon et la diversité des groupes socioculturels considérés. Plusieurs affections recensées dans cette étude ont été déjà mentionnées par des études antérieures comme susceptibles d'être soignées par C. millenii, tels l'ictère et l'abcès (Akoègninou et al., 2006), ou le paludisme (Adomou et al., 2012 ; Koudouvo et al., 2016). C. millenii est aussi une source de vitamine C pour contrer les infections (Olayiwola et al., 2013). Plusieurs parties de l'arbre sont utilisées en médecine traditionnelle, dont principalement les feuilles. La décoction est le mode de préparation le plus employé. Ces résultats sont semblables à ceux de Denou et al., (2016) et Koudouvo et al. (2016). La présence d'alcaloïdes, de tannins et de saponine dans la feuille (Sonibare et al., 2009) pourrait justifier son usage dans le traitement de plusieurs maladies. La décoction permet de recueillir au mieux les principes actifs et atténue ou annule l'effet toxique de certaines recettes médicinales (Salhi et al., 2010).

La fréquence de citation élevée du paludisme (53,9\%) résulte de l'étendue de cette maladie et de l'efficacité thérapeutique de la décoction des feuilles de $C$. millenii $(\mathrm{IF}=1)$.

\section{Relation entre groupes socioculturels et catégories d'usage}

Les usages de C. millenii sont dépendants des groupes socioculturels. Des observations similaires ont été faites par Sop et al. (2012) sur plusieurs plantes ligneuses au Burkina Faso. La diversité des utilisations peut être expliquée par les conditions environnementales locales qui gouvernent la distribution et la disponibilité de l'espèce (Sop et al., 2012). Elle peut être également due aux besoins des populations locales (Houètchégnon et al., 2015 ; Assogba et al., 2017).

\section{Relation entre groupes socioculturels, âge-genre et parties utilisées}

Les connaissances de l'utilisation des parties de $C$. millenii varient selon les groupes socioculturels comme l'ont par exemple rapporté les études de Wédjangnon et al. (2016) sur Mansonia altissima, de Assogba et al. (2017) sur Bombax costatum au Bénin, de Case et al. (2005) sur la flore locale en Papouasie-Nouvelle-Guinée, et de Franco et al. (2014) sur Koompassia excelsa en Malaisie. La variation des utilisations peut être expliquée non seulement par les différences culturelles mais aussi par la disponibilité locale d'autres espèces remplissant les mêmes fonctions (Houètchégnon et al., 2015).

Les connaissances de l'utilisation des parties de l'espèce varient également avec le genre et l'âge, conformément à Souto et Ticktin (2012), mais à l'encontre de De Caluwe et al. (2009) pour le baobab au Nord-Bénin. En effet, dans les ménages africains, ce sont généralement les femmes qui se chargent de l'approvisionnement en bois de feu pour la cuisson et le chauffage des aliments. Ceci pourrait expliquer le fait que les femmes préfèrent l'utilisation du tronc et des branches de $C$. millenii. La bonne connaissance des jeunes hommes sur l'utilisation de la pulpe viendrait du fait qu'ils en consomment souvent, surtout lorsqu'ils vont à la chasse. La connaissance particulière des hommes adultes sur l'utilisation de l'écorce pourrait résulter de leur forte implication dans la médecine traditionnelle.

\section{Commercialisation}

Au nombre des parties commercialisées, les feuilles et fruits se révèlent majoritaires. Ce résultat s'accorde avec ceux de Adomou et al. (2012), indiquant que la feuille de C. millenii est commercialisée dans le marché d'Abomey-Calavi au Bénin, mais aussi de Kokou et al. (2013), mentionnant la vente des feuilles sur la plupart des étalages de plantes médicinales dans les marchés de Lomé, au Togo. 
La commercialisation des autres parties n'est pas rapportée dans la littérature. La prédominance des feuilles peut s'expliquer par le fait qu'elles sont utilisées dans le traitement de plusieurs maladies (Odugbemi, 2006 ; Koudouvo et al., 2016 ; Houmenou et al., 2017).

\section{Menaces et stratégies endogènes de conservation}

L'étude révèle que la coupe de bois de chauffe, le défrichement à des fins agricoles, les feux de végétation, l'urbanisation et le prélèvement de diverses parties de la plante sont les principales menaces à l'égard de C. millenii, conformément à de précédents travaux sur les fruitiers sauvages (Balemie et Kebebew, 2006 ; Gouwakinnou et al., 2011 ; Fandohan et al., 2017).

C. millenii est conservée par très peu de personnes dans les champs et les agglomérations, ce qui ne s'accompagne que d'une faible tentative de domestication. L'usage fréquent d'une plante, sans domestication, augmente sa vulnérabilité (Keirungi et Fabricius, 2005). Or, plus le nombre de catégories d'usage d'une plante est élevé, plus elle est sollicitée et donc menacée (Ayèna et al., 2016). La maîtrise de la sylviculture de C. millenii est envisageable pour assurer sa domestication.

\section{Conclusion}

Cette étude montre que l'utilisation de Cola milllenii est diversifiée : médicinale, alimentaire, magique, brosse végétale, emballage, bois de service, bois énergie et bois d'œuvre. Les usages de l'espèce varient en fonction des groupes socioculturels. L'espèce est essentiellement utilisée à des fins alimentaires dans les groupes socioculturels situés dans la zone soudano-guinéenne du Bénin (Anii, Chabè). Trois tendances ont été observées chez les peuples situés dans la zone guinéenne du pays. Les uns lui accordent un intérêt médicinal (Aïzo) ; d'autres l'utilisent davantage comme brosse végétale, bois de service et pour ses propriétés magiques (Adja, Kotafon) ; d'autres encore la valorisent plus comme bois d'œuvre (Mahi).

La prise en compte de ces spécificités pourrait contribuer à l'élaboration d'une stratégie de gestion durable de l'espèce. Les feuilles et les fruits sont les parties les plus commercialisées. Malgré son importance, l'espèce subit diverses pressions et bénéficie de très peu de mesures de conservation. Des recherches futures devraient se pencher sur sa sylviculture pour assurer sa domestication.

\section{Remerciements}

Nous remercions la Fondation internationale pour la science pour avoir soutenu I. F. Lawin par l'octroi du Grant $n^{\circ}$ D/5875-1. A. B. Fandohan a bénéficié d'un soutien de la Fondation Alexander von Humboldt (Return fellowship $n^{\circ}$ 3.4, RKS, BEN/1155509 et Equipment Subsidy $n^{\circ}$ 3.6, BEN/1155509). Nous remercions également les évaluateurs pour leurs commentaires et corrections qui ont permis d'améliorer la qualité de cet article.

\section{Références bibliographiques}

Achigan-Dako E. G., Pasquini M. W., Assogba-Komlan F., N'danikou S., Yédomonhan H., Dansi A., Ambrose-Oji B., 2010. Traditional vegetables in Benin. Cotonou, Institut national des recherches agricoles du Bénin, Imprimeries du CENAP, 282 p.

Adomou A. C., 2005. Vegetation pattern and environmental gradients in Benin: Implications for biogeography and conservation. PhD thesis, Wageningen University, Wageningen, Netherlands, $133 \mathrm{p}$.

Adomou A. C., Yedomonhan H., Djossa B., Legba S. I., Oumorou M., Akoegninou A., 2012. Étude ethnobotanique des plantes médicinales vendues dans le marché d'Abomey-Calavi au Bénin. International Journal of Biological and Chemical Science, 6 (2): 745-772. http://dx.doi.org/10.4314/ijbcs.v6i2.18

Agbogidi O. M., 2010. Ethno-botanical survey of the non-timber forest products in Sapele Local Government Area of Delta State, Nigeria. African Journal of Plant Science, 4 (3): 183-189. http:// www.academicjournals.org/ajps

Akoègninou A., Van der burg W. J., Van der Maesen L. J. G., Adjakidjè V., Essou J. P., Sinsin, B., et al., 2006. Flore analytique du Bénin. Leiden, Pays-Bas, Backhuys Publishers, 1034 p.

Akpavi S., Woegan A. Y., Dourma M., Tozo K., Batawila K., Wala K., et al., 2011. Que sont devenues les plantes autrefois consommées par les divers groupes ethnoculturels du Togo ? Agronomie Africaine, 23 (2) : 147-160. https://www.ajol.info

Assogba G. A., Fandohan A. B., Salako V. K., Assogbadjo A. E., 2017. Usages de Bombax costatum (Malvaceae) dans les terroirs riverains de la Réserve de biosphère de la Pendjari, République du Bénin. Bois et Forêts des Tropiques, 333 : 17-29. https://doi. org/10.19182/bft2017.333.a31465

Assogbadjo A. E., Glèlè Kakaï R., Chadare F. J., Thomson L., Kyndt T., Sinsin B., et al., 2008. Folk classification, perception and preferences of baobab products in West Africa: consequences for species conservation and improvement. Economic Botany, 62 (1): 74-84. https://doi.org/10.1007/s12231-007-9003-6

Assogbadjo A. E., Glèlè Kakaï R., Vodouhê F. G., Djagoun C. A. M. S., Codjia J. T. C., Sinsin B., 2012. Biodiversity and socioeconomic factors supporting farmers' choice of wild edible trees in the agroforestry systems of Benin (West Africa). Forest Policy and Economics, 14: 41-49. http://doi.org/10.1016/j. forpol.2011.07.013

Atakpama W., Batawila K., Dourma M., Pereki H., Wala K. Dimobe K., Akpagana K., Gbeassor M., 2012. Ethnobotanical Knowledge of Sterculia setigera Del. in the Sudanian Zone of Togo (West Africa). International Scholarly Research Network, 2012: 1-8. http:// doi:10.5402/2012/723157

Ayèna A. C., Agassounon Djikpo Tchibozo M., Assogbadjo A. E., Agbangla C., Ahanhanzo C., 2016. Usages et vunérabilité de Pterocarpus santalinoides L'her. ex de (Papillionoidae), une plante utilisée dans le traitement des gastro-entérites dans le Sud du Bénin. European Scientific Journal, 12 (6). http://dx.doi. org/10.19044/esj.2016.v12n6p218

Balemie K., Kebebew F., 2006. Ethnobotanical study of wild edible plants in Derashe and Kucha Districts, South Ethiopia. Journal of Ethnobiology and Ethnomedicine, 2 (53): 1-9. http:// www.ethnobiomed.com/content/2/1/53

Campos M. T., Ehringhaus C., 2003. Plant virtues are in the eyes of the beholders: a comparison of known palm uses among indigenous and folk communities of south western Amazonia. Economic Botany, 57: 324-344. https://doi. org/10.1663/0013-0001(2003)057[0324:PVAITE]2.0.CO:2 
Case R. J., Pauli G. F., Soejarto D. D., 2005. Factors in Maintaining Indigenous Knowledge among Ethnic Communities of Manus Island. Economic Botany, 59: 356-365. https://doi. org/10.1663/0013-0001(2005)059[0356:FIMIKA]2.0.CO:2

Dagnélie P., 1998. Statistiques théoriques et appliquées. Bruxelles, Belgique, De Boeck, 517 p.

De Caluwe E., De Smedt S., Assogbadjo A. E., Samson R., Sinsin B., Van Damme P., 2009. Ethnic differences in use value and use patterns of baobab (Adansonia digitata L.) in northern Benin. African Journal of Ecology, 47 (3): 433-440. https://doi. org/10.1111/i.1365-2028.2008.01023.x

Denou A., Koudouvo K., Togola A., Aziati K. Y., Esseh K., Ajavon C. A., et al., 2016. Traditional knowledge on antimalarial plants having analgesic properties used in Togo Maritime Region. The Journal of Ethnobiology and Traditional Medicine, Photon, 126: 1160-1170. http://www.academia.edu/26038225/Traditional knowledge on antimalarial_plants having analgesic properties used in Togo Maritime Region

Djaha A. J. B., Gnahoua G. M., 2014. Contribution à l'inventaire et à la domestication des espèces alimentaires sauvages de Côte d'Ivoire: Cas des Départements d'Agboville et d'Oumé. Journal of Applied Biosciences, 78 : 6620-6629. https://www. ajol.info/index.php/jab/article/view/105831

Dossou M. E., Houessou G. L., Lougbégnon O. T., Tenté A. H. B., Codjia J. T. C., 2012. Étude ethnobotanique des ressources forestières ligneuses de la forêt marécageuse d'Agonvè et terroirs connexes au Bénin. Tropicultura, 30 (1) : 41-48. https://www.researchgate.net/profile/Laurent Houessou/ publication/259558319

Fandohan A. B., Chadare F. J., Gouwakinnou G. N., Tovissode C. F., Bonou A., Djonlonkou S. F. B., et al., 2017. Usages traditionnels et valeur économique de Synsepalum dulcificum au Sud-Bénin. Bois et Forêts des Tropiques, 332 (2) : 18-30. https://doi.org/10.19182/bft2017.332.a31330

Franco F. M., Ghani B. A. A., Hidayati S., 2014. Biocultural importance of the Tanying [Koompassia excelsa (Becc.) Taub.] tree for the Berawan of Loagan Bunut, Sarawak, Malaysia. Indian Journal of Traditional Knowledge, 13: 63-69. http://nopr.niscair.res. in/handle/123456789/26028

Gouwakinnou G. N., Lykke A. M., Assogbadjo A. E., Sinsin B., 2011. Local knowledge, pattern and diversity of use of Sclerocarya birrea. Journal of Ethnobiology and Ethnomedicine, 7 (8): 1-9. https://doi.org/10.1186/1746-4269-7-8

Houètchégnon T., Gbèmavo D. S. J. C., Ouinsavi C., Sokpon N., 2015. Ethnobotanical knowledge and traditional management of african mesquite (Prosopis africana Guill., Perrot. et Rich.) populations in Benin, West Africa. The Journal of Ethnobiology and Traditional Medicine, Photon, 125: 1124-1135. http:/ dx.doi.org/ 10.1155/2015/101373

Houmenou V., Adjatin A., Tossou M. G., Yedomonhan H., Dansi A., Gbenou J., et al., 2017. Étude ethnobotanique des plantes utilisées dans le traitement de la stérilité féminine dans les départements de l'Ouémé et du plateau au Sud Bénin. International Journal of Biological and Chemical Sciences, 11 (4) : 1851-1871. http://dx.doi.org/104314/ijbcs.v11i4.34 Keirungi J., Fabricius C., 2005. Selecting medicinal plants for cultivation at Nqabara on the Eastern Cape Wild Coast, South Africa. South African Journal of Sciences, 101: 497-501. https:// hdl.handle.net/10520/EJC96329

Kokou K., Batawila K., Akpagana K., Tossou M. G., Akoegninou A., 2013. Impact écologique de la cueillette et commercialisation des plantes médicinales au Togo. www.glinus.com/2013/12/ impact-ecologique-de-la-cueillette-et.html
Kouamé N. M. T., Gnahoua G. M., Kouassi K. E., Traoré D., 2008. Plantes alimentaires spontanées de la région du Fromager (Centre-Ouest de la Côte d'Ivoire) : flore, habitats et organes consommés, Science et Nature, 5 (1) : 61-70. http://dx.doi. org/10.4314/scinat.v5i1.42152

Koudouvo K., Esseh K., Denou A., Aziati T., Ajavon C., Afanyibo Y.-G., et al., 2016. Étude ethnopharmacologique des recettes médicinales antipaludiques du Togo pour la formulation d'un phytomédicament de prise en charge du paludisme. Bulletin de la Recherche Agronomique du Bénin, 79 : 54-70. http:// www.slire.net/download/2372/article 6 complet brab 79 juin 2016 koudouvo et al tude ethno-pharmacologique.pdf Moupela C., Vermeulen C., Daïnou K., Doucet J.-L., 2011. Le noisetier d'Afrique (Coula edulis Baill.). Un produit forestier non ligneux méconnu. Biotechnologie, Agronomie, Société et Environnement, 15 (3) : 485-495. https://popups.uliege.be/17804507/index.php?id=7807

Odugbemi T., 2006. Outlines and Pictures of Medicinal Plants from Nigeria. Vol. 10. University of Lagos Press, 158 p.

Olayiwola I. O., Akinfenwa V. O., Oguntona C. O., Sanni S. A., Onabanjo O. O., Afolabi W. A. O., 2013. Phytonutrient, antioxidant and mineral composition of some wild fruits in South West Nigeria. Nigeria Food Journal, 31 (2): 33-40. https://doi. org/10.1016/S0189-7241(15)30074-6

Pilgrim S., Smith D., Pretty J., 2007. A cross regional assessment of the factors affecting ecoliteracy. Implications for policy and practice. Ecological Applications, 17 (6): 1742-1751. https:// doi.org/10.1890/06-1358.1

Salhi S., Fadli M., Zidane L., Douira A., 2010. Études floristique et ethnobotanique des plantes médicinales de la ville de Kénitra (Maroc). Lazaroa, 31: 133-146. http://www.ethnopharmacologia.org/prelude2018/pdf/biblio-s3-nord-salhi.pdf

Sonibare M. A., Soladoye M. O., Sonibare O. O., 2009. Phytochemical and antimicrobial studies of four species of Cola Schott \& Endl. (Sterculiaceae). African Journal of Traditional, Complementary and Alternative Medicines, 6 (4): 518-525. https:// www.ajol.info/index.php/ajtcam/article/view/57182

Sop T. K., Oldeland J., Bognounou F., Schmiedel U., Thiombiano A., 2012. Ethnobotanical knowledge and valuation of woody plants species: a comparative analysis of three ethnic groups from the sub-Sahel of Burkina Faso. Environment, Development and Sustainability, 14: 627-649. https://doi.org/10.1007/ s10668-012-9345-9

Souto T., Ticktin T., 2012. Understanding interrelationships among predictors (age, gender, and origin) of local ecological knowledge. Economic Botany, 66 (2): 149-164. https://doi. org/10.1007/s12231-012-9194-3

Tardio J., Pardo-De-Santayana M., 2008. Cultural importance indices: a comparative analysis based on the useful wild plants of southern Cantabria (northern Spain). Economic Botany, 62 (1): 24-39. https://doi.org/10.1007/s12231-007-9004-5 Wédjangnon A. A., Houètchégnon T., Ouinsavi C., 2016. Caractéristiques ethnobotaniques et importance socio-culturelle de Mansonia altissima A. Chev. au Bénin, Afrique de l'Ouest. Journal of Animal and Plant Sciences, 29 (3) : 4678-4690. http:// www.m.elewa.org/JAPS

Bois et Forêts des Tropiques - Revue scientifique du Cirad
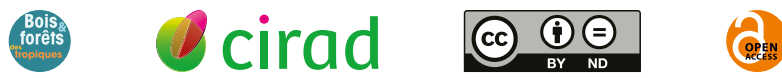

Cirad - Campus international de Baillarguet, 34398 Montpellier Cedex 5, France - Contact : bft@cirad.fr - ISSN : L-0006-579X 